Case Report

\title{
Radiotherapy and Hyperthermia in the Treatment of Locoregional Recurrent Cancer-Report of the First Patients Treated in Portugal
}

\author{
Maria Adelina Costa ${ }^{1,}$,, Carlos Fardilha ${ }^{1}$, Graça Fonseca ${ }^{1}, J_{0 a o}$ Conde $^{1}$, Carmen Calcada $^{1}$, \\ Fatima Rodrigues ${ }^{1}$, Paula Genesio ${ }^{1}$, Fernanda Ponte ${ }^{1}$, Joana Vale ${ }^{1}$, Guilherme Campos ${ }^{1}$, \\ Carla Seixas $^{1}$, Andre Ferreira ${ }^{2}$, Carla Sousa ${ }^{2}$, Moreira Pinto ${ }^{2}$, Paulo Costa ${ }^{1}$ \\ ${ }^{1}$ Radiation Oncology Department Julio Teixeira, CUF Institute, Porto, Portugal \\ ${ }^{2}$ Medical Oncology Service, Hospital CUF, Porto, Portugal \\ Email address: \\ adelinacosta@sapo.pt (M. A. Costa), carlosfardilha@yahoo.com (C. Fardilha), gracafonseca@gmail.com (G. Fonseca), \\ macaconde@hotmail.com (J. Conde), carmen.calcada@iol.pt (C. Calçada), fatimaeirarodrigues@gmail.com (F. Rodrigues), \\ pmgenesio@gmail.com (P. Genesio), fmmponte@gmail.com (F. Ponte), joana.vale@gmail.com (J. Vale), \\ guilherme.campos.03@gmail.com (G. Campos),cmatss@gmail.com (C. Seixas), andre.a.ferreira@jmellosaude.pt (A. Ferreira), \\ carla.sousa@jmellosaude.pt (C. Sousa), amoreiap@gmail.com (M. Pinto),paulo.serafim@gmail.com (P. Costa) \\ ${ }^{*}$ Corresponding author
}

\section{To cite this article:}

Maria Adelina Costa, Carlos Fardilha, Graça Fonseca, Joao Conde, Carmen Calcada, Fatima Rodrigues, Paula Genesio, Fernanda Ponte, Joana Vale, Guilherme Campos, Carla Seixas, Andre Ferreira, Carla Sousa, Moreira Pinto, Paulo Costa. Radiotherapy and Hyperthermia in the Treatment of Locoregional Recurrent Cancer-Report of the First Patients Treated in Portugal. Science Journal of Clinical Medicine. Vol. 8, No. 1, 2019, pp. 1-5. doi: 10.11648/j.sjcm.20190801.11

Received: March 26, 2019; Accepted: May 6, 2019; Published: May 31, 2019

\begin{abstract}
Background and purpose: The association of hyperthermia with radiotherapy has been shown to be a valid approach in the treatment of locoregional recurrence of previously irradiated malignant tumors. The purpose of the present study is to describe the initial experience of the Radiation Oncology Department Júlio Teixeira SA (CUF Institute) and the Medical Oncology Service (Hospital CUF Porto) in the treatment of these lesions, reporting the first cases treated in Portugal with this therapeutic approach. Material and methods: The authors performed a retrospective analysis of the 18 patients, 16 females and 2 males, with unresectable tumor recurrences, in previously irradiated areas, treated with radiotherapy associated with hyperthermia between May 2016 and March 2017, totalizing 25 treatments. Breast disease was the most frequent, accounting for $72 \%$ of cases. Results: The median follow-up period was 4,5 months. The complete response rate was $44 \%$. A favorable response was observed in all treatments, with decrease in pain, bleeding and infection, with and no grade 3 toxicities. Conclusions: The results are encouraging, with improved quality of life and patient self-esteem with acceptable toxicity. The combined treatment of hyperthermia and radiotherapy seems to be a valid option in the local control of recurrent unresectable neoplasms at previously irradiated sites, whose therapeutic options are limited.
\end{abstract}

Keywords: Radiotherapy, Hyperthermia, Locoregional Recurrence, Reirradiation

\section{Introduction}

If not managed, unresectable locoregional recurrences can cause serious suffering to the affected patients. They are responsible for severe pain, with bleeding and/or local infection, in lesions which may vary in appearance. [1, 2]. For the patient, noticing the visible uncontrolled tumoral growth with the above-mentioned characteristics, constitutes an extremely painful living experience, both physically and psychologically. It is not difficult to understand, therefore, how challenging these situations can be for clinicians when facing the limitations of effective therapeutic approaches. 
Radiotherapy, often regarded as the salvation therapy, when delivered in previously irradiated regions, can lead to an unacceptable level of local toxicity if effective doses are preconized [1-4].

The treatment combining radiotherapy (RT) and hyperthermia (HT) results in a complementary tumoricidal effect and it has been used to treat these of lesions [3-14].

The TCS 42 System is a non-invasive medical equipment of local HT that, through the use of its two active electrodes, emits a radiofrequency of 13.56 MHZ. This allows to raise the tumoral temperature, focally and selectively (Figures 1 and 2).

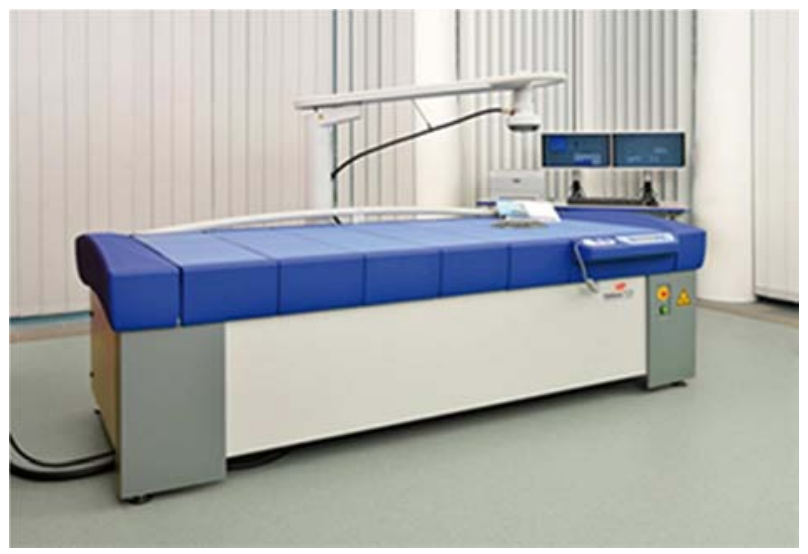

Figure 1. TCS 42 System.

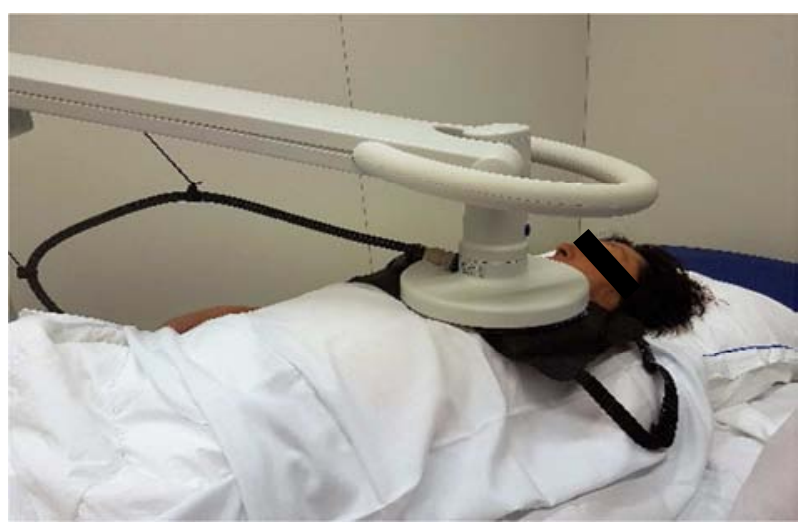

Figure 2. Treatment.

The authors describe the initial experience in Portugal, in the treatment of locoregional recurrence of malignant tumors, with the use of radiotherapy (reirradiation) in association with local hyperthermia, with a special focus on the initial tumoral response and associated acute toxicities.

\section{Material and Methods}

A retrospective analysis of 18 patients, 16 females and 2 males, with unresectable recurrent tumors, in previously irradiated areas, treated with RT associated to HT, in the period between May 2016 and March 2017, totaling 25 treatments, was performed.

In all cases, an intensity modulated radiation therapy (IMRT) technique was used and all patients were submitted to the same treatment regimen (Figure 3).

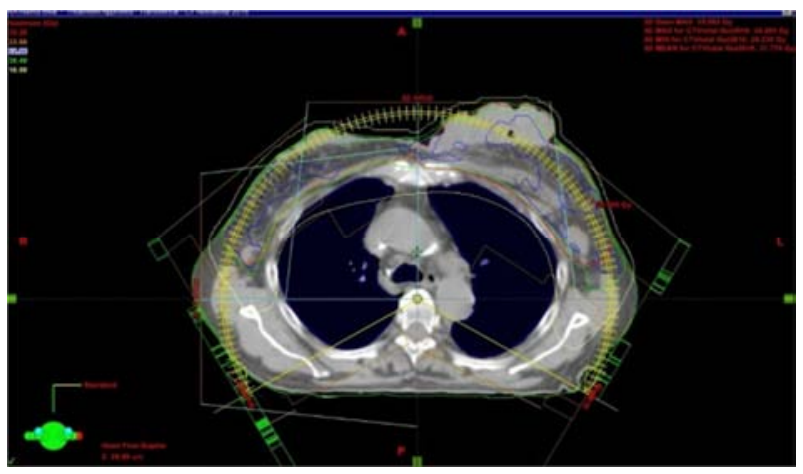

Figure 3. Radiation therapy treatment planning.

The treatment consisted in delivering a total radiation dose of 32 Gy, 4 Gy per fraction, totaling 8 fractions, delivered twice a week (an interval of 3 days between fractions), for 4 weeks, with $6 \mathrm{MV}$ photons. An HT treatment per week with the duration of 1 hour was added to the RT. The interval between RT and HT was 30 minutes. The TCS 42 System was used, emitting a radiofrequency of $13.56 \mathrm{MHz}$ through 2 active electrodes.

Before the RT/HT treatment, $28 \%$ of the patients presented infected lesions and in $12 \%$ of them the lesions presented an active bleeding. Twenty eight $\%$ of the patients were undergoing a systemic treatment (hormonal in 16\%, target therapy in $8 \%$ and both in $4 \%$ ), which was maintained during $\mathrm{RT} / \mathrm{HT}$.

The acute events were assessed against the CTCAE 4.03 scale.

\section{Results}

The median age at diagnosis was 62 years (range 42-79). Breast pathology was the most frequent, representing $72 \%$ of all cases, followed by head and neck (12\%), gynecological $(8 \%)$, brain (4\%) and endocrine tumors (4\%) (Table 1$)$.

Table 1. Patient and Tumor-related characteristics at the time of combined treatment

\begin{tabular}{lll}
\hline Mean (range) & n & \% \\
\hline Age (yrs) & $62(42-79)$ & \\
Interval between initial diagnosis and relapse (months) & $50(80-120)$ & \\
Interval between initial radiotherapy and re-irradiation & $55(70-128)$ & \\
(months) & & 50 \\
Presence of distant metastasis & 9 & \\
Anatomic site of current recurrence & & 72 \\
Breast / Chest wall & 11 & 12 \\
Head and neck & 3 & 8 \\
Vulva & 2 & 4 \\
Brain & 1 & \\
Endocrinic system & 1 & \\
Dose of radiotherapy previously administrated (Gy) & $48(50-60)$ & \\
\hline
\end{tabular}

The skin reaction varied from grade 1 erythema to moist desquamation, treated with conservative measures. Some examples of the treated patients are shown in Figures 4 to 11 . 


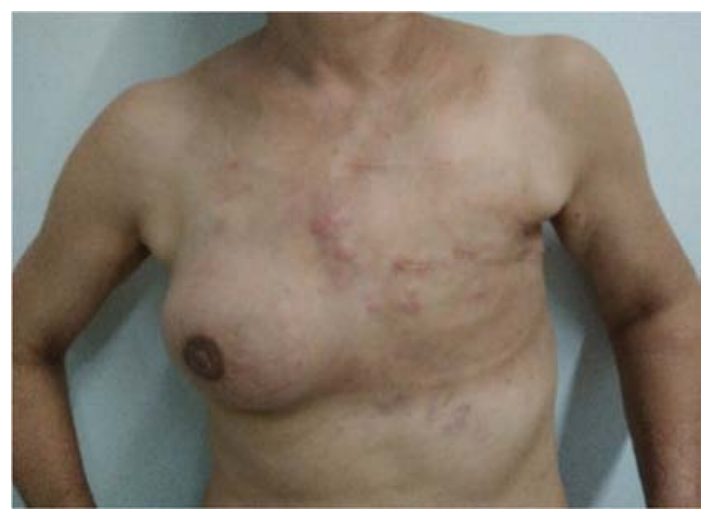

Figure 4. Before treatment.

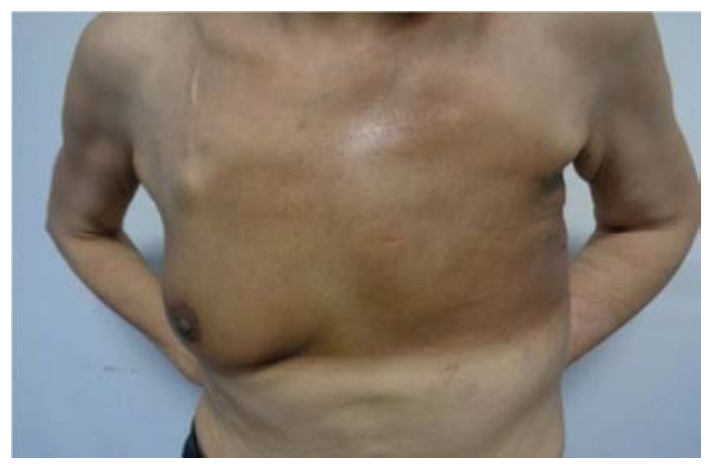

Figure 5. Three months later.

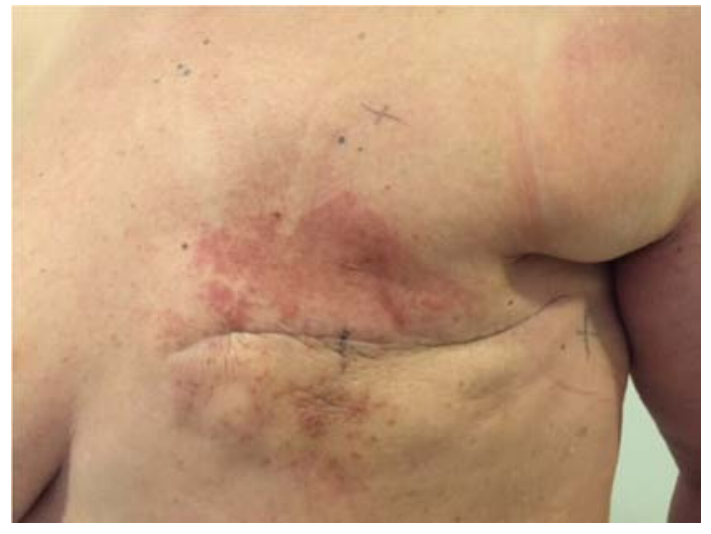

Figure 6. Before treatment.

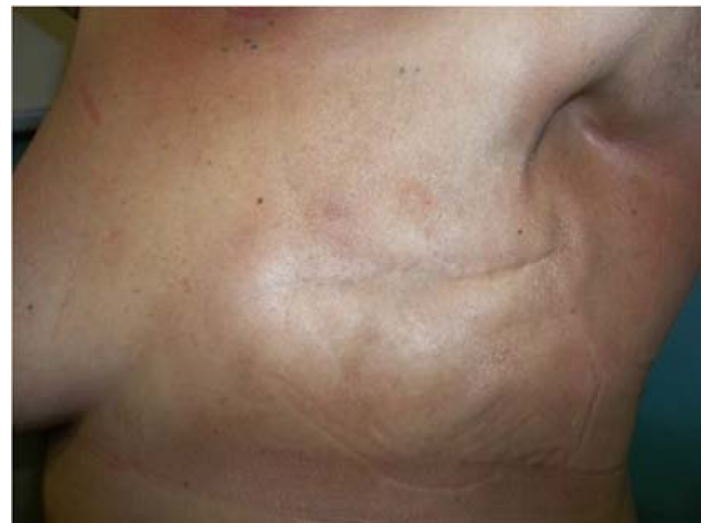

Figure 7. Three months later.

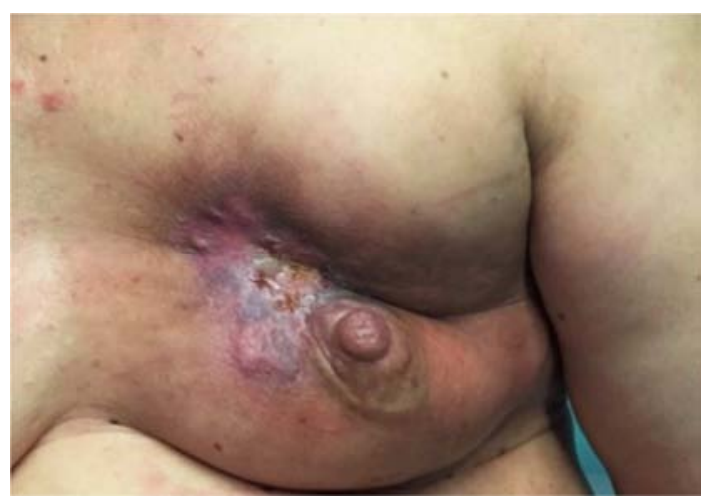

Figure 8. Before treatment.

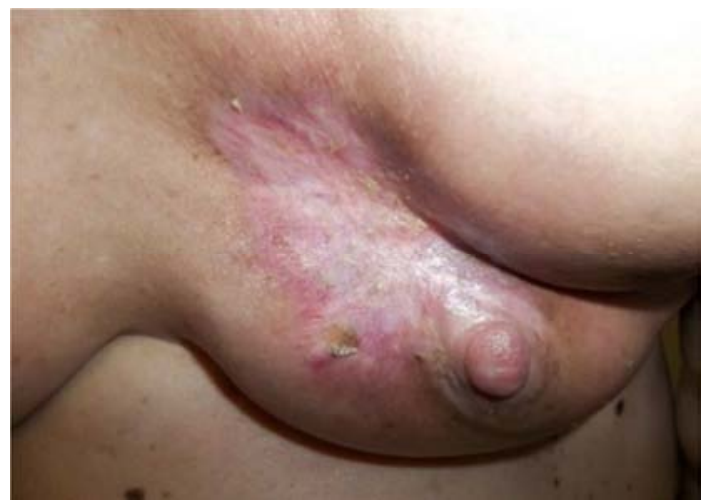

Figure 9. Three months later.

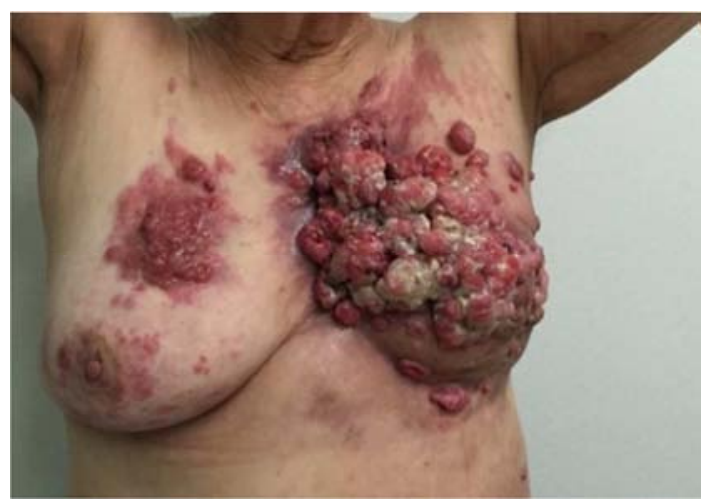

Figure 10. Before treatment.

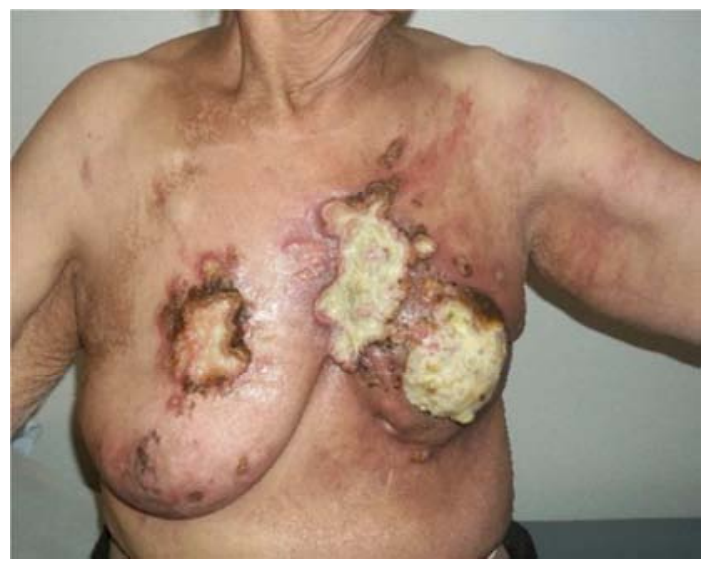

Figure 11. Three months later. 
No grade 3 toxicities were observed. Grade 2 radiodermatitis occurred in $32 \%$ of the cases; for the remaining patients radiodermatitis was grade 1 .

Intolerance to hyperthermia was observed in $8 \%$ of the cases, caused by pain and inability to maintain the treatment position.

There was a complete response in all cases submitted to systemic therapy. Three patients presented disease progression outside of the treatment fields, and in one case there was a recurrent lesion in the irradiated area, 2 months after finalizing $\mathrm{RT} / \mathrm{HT}$.

With an average follow-up period of 4.5 months (range 1-18 months), $44 \%$ of the cases had no clinical evidence of disease and we had a partial response in $56 \%$ of the patients with reduction of pain, bleeding and infection.

\section{Discussion}

Reirradiating these patients with a radiation dose of $32 \mathrm{~Gy}$, with concomitant HT, led to a complete response in $44 \%$ of the cases. The same RT scheme, 8 fractions of $4 \mathrm{~Gy}$, without HT was used in the study RTOG 81-04, which reported a complete response rate of $26 \%$ [4]. The contribution of HT in combination with RT was confirmed by a randomized study from the International Collaborative Hyperthermia Group, in 1996 [5]. The J van der Zeel et al study reported a complete response rate of $71 \%$ with the same treatment scheme [1].

Despite the decades of research, there is no consensus yet about the exact mechanism of cellular death induced by hyperthermia, but the combination of necrosis induced by heat and the protein inactivation, like the reparation enzymes, is the most likely hypothesis [3, 12-18]. The inactivation of these enzymes, by inhibiting the reparation of sub-lethal damages in the DNA, boosts one of the key-mechanisms of cellular death induced by radiation. This HT action mechanism works like a complementary action to RT. Other HT effects were identified, such as changes in the tumoral cytoskeleton and in the membrane structures [3, 6, 7-13].

HT damages the cells and increases the sensitivity to RT as a function of temperature and duration of the treatment $[3$, 14-18].

As temperature and duration increases, the cellular death rate also increases. For short treatments in well vascularized tissue at a moderate temperature $\left(<42^{\circ} \mathrm{C}\right)$, HT may increase the tumor perfusion, making it more susceptible to RT and increasing cellular death [2, 3, 11-15].

This effect is further enlarged by the inactivation of enzymes engaged in aerobic metabolism, reducing the oxygen consumption and increasing tumoral oxygenation [3, 16-18].

At temperatures above $42^{\circ} \mathrm{C}$, tumor revascularization is damaged, resulting in a reduced blood flow and hypoxia. We add then another complementary effect of RT and HT, since the cells sensitivity to ionizing radiation is influenced by its stage in the cellular cycle $[3,8,12-16]$.

The cells in stages G0 and S are less sensitive to damage caused by RT but show higher sensitivity to HT. Hence, HT is not a simple way of delivering more oxygen to a tumor in order to boost the RT. Also, it is a much more complex process involving multiple mechanisms [3, 11-15].

Cancer cells are especially vulnerable to heat. In vivo studies show that temperatures in the range $40-44^{\circ} \mathrm{C}$ cause more selective damages to tumoral cells. One main factor in the difference between normal cells and tumoral cells in terms of HT sensitivity can be explained by basic physiological differences between the vascularization of tumoral and normal tissue $[3,8,9]$.

The vascularization of normal tissues follows an efficient network of arteries, capillaries and veins, while the blood vessels of tumoral tissues are arranged in an anarchical way and are often ineffective and/or insufficient [1, 3, 12-17].

This abnormal vascularization results in a weak tumoral perfusion, with the creation of hypoxia and limiting the effectiveness of traditional RT. Other mechanisms that cause HT to generate a selective cytotoxic effect in tumoral cells include the induction of apoptosis and the suppression of cells proliferation of tumor stem cells [1, 2, 13-16].

HT has been showing effectiveness in the treatment of a wide range of neoplasias, including head and neck, breast, brain, bladder, cervix, rectum, lung, esophagus, vulva and vaginal tumors, as well as skin tumors (melanoma and non-melanoma) and sarcomas [1, 2, 6, 8, 9, 10, 17, 18].

Similarly to other cancer treatments, the tolerance of normal tissues to HT is also variable and different disease locations require specific thermic doses. The central nervous system tissues are particularly sensitive, and may suffer irreversible damages with treatments longer than 40 minutes and at temperatures between $42-42.5^{\circ} \mathrm{C}$. However, most normal tissues show little or no permanent damage with 1 -hour treatments up to $44^{\circ} \mathrm{C}[1,3,10,11,14,18]$.

Although it has not been established that the local control of this type of recurrent tumors with the mentioned therapy association influences the global survival rate, the absence of symptomatic and visible lesions results in an improvement in the quality of life for the patients $[1,3,17,18]$.

\section{Conclusions}

Hyperthermia is one of the hot topics that have been discussed over decades and must therefore be considered of general interest.

The basic rationale of the heat to enhance the effect of radiation is evident.

Over the years, several prospective and randomized studies have emerged demonstrating a clear benefit of combined HT and RT for multiple entities, such breast cancer recurrences, cervix carcinoma or head and neck cancers.

Reirradiation combined with local hyperthermia in the treatment of recurrent disease is increasingly becoming a treatment option for these patients, particularly in cases where, apparently, there are no other valid therapeutic options.

It acquires then a significant importance as another hope in selected cases, with reassuring results with low doses of radiotherapy, which is particularly important in these cases, previously irradiated. The systemic therapy seems to leverage 
the effects of RT combined with HT. We observed a complete response in all cases submitted to systemic therapy.

At the time of the present analysis, $44 \%$ of the cases had no clinical evidence of disease and we had a partial response in $56 \%$ of the patients with reduction of pain, bleeding and infection.

Add to this the fact that the treatment is well-tolerated and the toxicity is acceptable, and we can consider this promising therapeutic approach will tend to be more used, also for its justified palliation, for improving greatly the quality of life and the self-esteem of the patients.

Through continuous improvement of HT delivery, planning, and monitoring techniques, treatment effects may further improve.

A longer follow-up period will be required in order to assess late reactions and the remission duration of these tumors.

\section{References}

[1] J Zee, B Holt, PJM Rietveld, et al. Reirradiation combined with hyperthermia in recurrent breast cancer results in a worthwhile local palliation. British Journal of cancer (1999) 79 (3/4), 483-490.

[2] NG Huilgol, S Gupta, CR Sridhar. Hyperthermia with radiation in the treatment of locally advanced head and neck cancer: a report of randomized trial. J. Cancer Res. Ther. (2010) 6 (4), 492-496.

[3] M Mallory, E Gogineni, GC Jones, et al. Therapeutic hyperthermia: The old, the new, and the upcoming. Criticals Reviews in Oncology/Hematology 97 (2016), 56-64.

[4] CA Perez, B Gillespie, T Pajak, et al. Quality assurance problems in clinical hyperthermia and their impact on therapeutic outcome: a Report by the Radiation Therapy Oncology Group. Int J Radiat Oncol Biol Phys (1989) 16(3), 551-8.

[5] International Collaborative Hyperthermia Group. Hyperthermia in the treatment of superficial localized primary and recurrent breast cancer - results from five randomized controlled trials. Int J Radiat Oncol Biol Phys (1996) 35, 731-744.

[6] H Sahinbas. Retrospective clinical study for advanced brain gliomas by adjuvant electro-hyperthermia treatment. Cancer Therapy (2012) 8, 139-149.

[7] HP Kok, J Crezee. A comparison of the heating characteristics of capacitive and radiative superficial hyperthermia. Int $\mathrm{J}$ hyperthermia (2017) 33(4), 378-386.
[8] NR Datta, S Rogers, SG Ordóñez, et al. Hyperthermia and radiotherapy in the management of head and neck cancers: A systematic review and meta-analysis. Int J Hyperthermia (2016) 32(1), 31-40.

[9] NR Datta, E Puric, D Klingbiel, et al. Hyperthermia and radiotherapy in locoregional recurrent breast cancers: A systematic review and meta-analysis. Int J Radiat Oncol Biol Phys (2016) 94(5), 1073-87.

[10] NR Datta, SG Ordóñez, US Gaipl, et al. Local hyperthermia combined with radiotherapy and/or chemotherapy: recent advances and promises for the future. Cancer Treat Rev (2015) 41(9), 742-53.

[11] NR Datta, E Puric, D Klingbiel, et al. Hyperthermia and radiation therapy in locoregional recurrent breast cancers: a systematic review and meta-analysis. Int J Radiat Oncol Biol Phys (2016) 94(5):1073-87.

[12] NR Datta, S Rogers, D Klingbiel, et al. Hyperthermia and radiotherapy with or without chemotherapy in locally advanced cervical cancer: a systematic review with conventional and network meta-analyses. Int J Hyperthermia (2016) 6736:1-34.

[13] LCHW Lutgens, PCM Koper, JJ Jobsen, et al. Radiation therapy combined with hyperthermia versus cisplatin for locally advanced cervical cancer: results of the randomized RADCHOC trial. Radiother Oncol (2016) 120(3):378-82.

[14] NR Datta, S Rogers, SG Ordonez, et al S. Hyperthermia and radiotherapy in the management of head and neck cancers: a systematic review and meta-analysis. Int J Hyperthermia (2016) 32(1):31-40.

[15] C Zhao, J Chen, B Yu, et al. Improvement in quality of life in patients with nasopharyngeal carcinoma treated with non-invasive extracorporeal radiofrequency in combination with chemoradiotherapy. Int J Radiat Biol (2014) 90(10):8538.

[16] M Kang, WQ Liu, YT Qin, et al. Long-term efficacy of microwave hyperthermia combined with chemoradiotherapy in treatment of nasopharyngeal carcinoma with cervical lymph node metastases. Asian Pac J Cancer Prev (2013) 14(12):7395400 .

[17] Linthorst M, Baaijens M, Wiggenraad R, et al. Local control rate after the combination of re-irradiation and hyperthermia for irresectable recurrent breast cancer: Results in 248 patients. Radiotherapy and Oncology (2015) 117: 217-222.

[18] Peeken JC, Vaupel P, Combs SE. Integrating hyperthermia into modern radiation oncology: what evidence is necessary? Frontiers in Oncology (2017) 7(132): 1-12. 